\title{
Effects of Switching from Liraglutide or Dulaglutide to Subcutaneous Semaglutide on Glucose Metabolism and Treatment Satisfaction in Patients with Type 2 Diabetes: Protocol for a Multicenter, Prospective, Randomized, Open-Label, Blinded-Endpoint, Parallel- Group Comparison Study (The SWITCH-SEMA 1 Study)
}

\author{
Hiroshi Nomoto · Chiho Oba-Yamamoto · Yuka Takahashi · Jun Takeuchi · So Nagai • \\ Hiroki Yokoyama · Shinji Taneda · Yoshio Kurihara · Shin Aoki · Hiraku Kameda • \\ Kyu Yong Cho $\cdot$ Akinobu Nakamura · Tatsuya Atsumi $\cdot$ Hideaki Miyoshi
}

Received: November 9, 2020 / Accepted: December 15, 2020 / Published online: January 24, 2021

(c) The Author(s) 2021

\begin{abstract}
Introduction: Glucagon-like peptide (GLP)-1 receptor agonists exert potent hypoglycemic effects in patients with type 2 diabetes (T2D) in a blood glucose concentration-dependent manner. Once-weekly subcutaneous administration of the GLP-1 receptor agonist semaglutide has beneficial effects on glycemic and body weight control, but it is currently unclear if
\end{abstract}

H. Nomoto · Y. Takahashi · H. Kameda ·

K. Y. Cho · A. Nakamura - T. Atsumi · H. Miyoshi $(\square)$

Department of Rheumatology, Endocrinology and Nephrology, Faculty of Medicine and Graduate School of Medicine, Hokkaido University, Sapporo, Japan

e-mail: hmiyoshi@med.hokudai.ac.jp

C. Oba-Yamamoto $\cdot$ J. Takeuchi

Sapporo Diabetes and Thyroid Clinic, Sapporo, Japan

\section{S. Nagai}

Division of Diabetes and Endocrinology,

Department of Medicine, Sapporo Medical Center,

NTT East Corporation, Sapporo, Japan

\section{H. Yokoyama}

Department of Internal Medicine, Jiyugaoka

Medical Clinic, Obihiro, Japan semaglutide provides superior glycemic control compared to conventional GLP-1 receptor agonists in the Japanese population. We aim to compare the effects of once-weekly subcutaneous semaglutide with those of liraglutide or dulaglutide administration in Japanese patients with T2D.

Methods: This study is a multicenter, prospective, randomized, open-label, blinded-endpoint, parallel-group trial. In total, 100 participants with T2D who have been treated

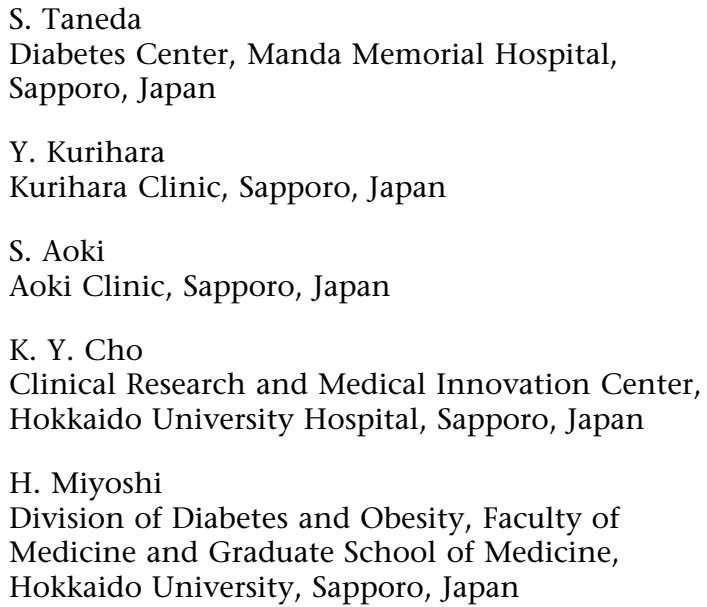


with liraglutide $(0.9-1.8 \mathrm{mg} /$ day in plan A) or dulaglutide (0.75 mg/week in plan B) for more than 12 weeks and have a glycated hemoglobin (HbA1c) level of $6.0-9.9 \%$ and a body mass index (BMI) of $\geq 22 \mathrm{~kg} / \mathrm{m}^{2}$ will be randomized to either continue using their existing GLP-1 receptor agonist or switch to subcutaneous semaglutide once weekly for 24 weeks. Biochemical analysis, physical assessment, and a quality-of-life questionnaire (DTSQ) will be completed at baseline and at the end of the study. The primary endpoint is the effect of semaglutide on the change in HbA1c. The secondary endpoints are the mean changes in total DTSQ score, body mass, abdominal circumference, systolic and diastolic blood pressure, pulse rate, factors associated with improvement in HbA1c and secondary endpoints, side effects, and other laboratory parameters.

Planned Outcomes: The results of the study will provide useful information regarding the effects of switching to semaglutide from other GLP-1 receptor agonists on glycemic control in patients with T2D.

Ethics and Dissemination: The Hokkaido University Certified Review Board (CRB no. 1180001) has approved the protocol (no. 018-005). The results will be disseminated in peer-reviewed journals and at scientific conferences.

Trial Registration: UMIN000042369 in the University Hospital Medical Information Network (UMIN); jRCT1011200008 in the Japan Registry of Clinical Trials (jRCT); pre-results.

Keywords: Glucagon-like peptide-1 receptor agonist; Treatment satisfaction; Type 2 diabetes mellitus

\section{Key Summary Points}

This randomized controlled study will be the first to directly compare the glycemic control and treatment satisfaction achieved with a switch to semaglutide administration to those achieved by continuing with other GLP-1 receptor agonists in Japanese patients with type 2 diabetes.
The study will make two different comparisons: plan A, a comparison of semaglutide and liraglutide; and plan $\mathrm{B}$, a comparison of semaglutide and dulaglutide.

The study is a multicenter, prospective, randomized, blinded-endpoint, parallelgroup trial, although participants will not be blinded to their treatment.

The study will be conducted at seven medical centers in a standard clinical practice setting, and will incorporate broad eligibility criteria, reflecting the real world.

\section{DIGITAL FEATURES}

This article is published with digital features, including a summary slide, to facilitate understanding of the article. To view digital features for this article go to https://doi.org/10.6084/ m9.figshare.13379093.

\section{INTRODUCTION}

One of the most important goals in the treatment of diabetes is the prevention and management of atherosclerotic diseases and diabetic complications, which is achieved by maintaining metabolic factors such as glucose and lipid metabolism, blood pressure (BP), and obesity within appropriate limits [1-3]. However, it is sometimes difficult to obtain good glycemic control using conventional therapies in daily clinical practice, even though reducing glycated hemoglobin (HbA1c) levels helps to reduce diabetic complications [3, 4]. In addition, some antihyperglycemic drugs cause hypoglycemia, leading to an increased risk of cardiovascular events, ultimately reducing quality of life (QOL) $[5,6]$. Certain antihyperglycemic agents require frequent administration because of their short duration of action, resulting in poor treatment compliance and QOL $[7,8]$. The ideal 
antidiabetic agent would have a potent hypoglycemic effect and a long duration of action but would not cause hypoglycemia.

Glucagon-like peptide (GLP)-1 receptor agonists such as liraglutide, exenatide, and dulaglutide have strong hypoglycemic effects that are exerted in a blood glucose concentration-dependent manner and do not tend to cause hypoglycemia [9]. In addition, these agents can reduce excess body weight by inhibiting gastric peristalsis and reducing appetite $[10,11]$. Most importantly, recent clinical trials have shown that they also have beneficial effects on cardiovascular outcomes $[12,13]$. Therefore, these agents are now widely prescribed. The frequency of administration of a GLP-1 receptor agonist depends on its half-life. As would be predicted, once-weekly administration of dulaglutide is associated with better QOL than once-daily liraglutide administration [14].

Recently, the newly approved once-weekly subcutaneous GLP-1 receptor agonist semaglutide was reported to have potent effects on hyperglycemia and body weight and to reduce cardiovascular risk compared to a placebo [15]. However, it is not known whether subcutaneous semaglutide administration yields superior results to conventional GLP-1 receptor agonists with respect to glycemic control and treatment satisfaction, especially in the Japanese population. In this prospective, randomized, open-label, parallel-group trial, we will compare the effects of once-weekly subcutaneous semaglutide administration to those of liraglutide and dulaglutide with respect to glycemic control and QOL in patients with type 2 diabetes (T2D).

\section{METHODS}

\section{Study Design}

This is a multicenter, open-label, prospective, randomized, blinded-endpoint, parallel-group comparison study that aims to compare the effects of the once-weekly subcutaneous GLP-1 receptor agonist semaglutide with those of liraglutide $0.9-1.8 \mathrm{mg} /$ day or dulaglutide
$0.75 \mathrm{mg} /$ week on glycemic control. Following enrollment and the provision of written informed consent (period 1), the participants will undergo serum and urine analyses and a physical examination to obtain baseline data. At the same time, they will complete the Diabetes Treatment Satisfaction Questionnaire (DTSQ), which is a self-administered questionnaire that records patient-reported outcomes [16]. At each study visit, clinic BP, pulse rate, body mass, and abdominal circumference will be measured. After the initial assessment, the participants will be randomized to either continue with their existing GLP-1 receptor agonist or to switch to once-weekly semaglutide administration. The randomization and allocation of the participants will be performed by an automated system that is independent of the participating sites (NorthNet; https://crmic. huhp.hokudai.ac.jp/page/?content=31). Participants in the semaglutide group will follow a semaglutide dose-escalation regimen (the maintenance dose of $0.5-1.0 \mathrm{mg}$ will be reached after 4 weeks of $0.25 \mathrm{mg}$ semaglutide administration once weekly). Semaglutide will be administered into the subcutis of the abdominal wall, thigh, or upper arm on the same day of each week. The treatments will be supervised through the appropriate medical care center for 24 weeks. All the participants will be encouraged to continue their diet and exercise therapy during the study. It will be possible to adjust the doses of hypoglycemic agents other than GLP-1 receptor agonists, such as sulfonylureas, glinides, and insulin, to avoid hypoglycemia, based on the recommendations of the Japan Diabetes Society. The doses of the other antihyperglycemic agents that are being administered concomitantly will not be adjusted during the study period. After 24 weeks of treatment, the serum and urine measurements and physical examination carried out at baseline will be performed again (period 3) (Fig. 1).

\section{Sample Selection}

Japanese patients with T2D will be enrolled if they are aged 20-89 years, have a HbA1c level of $6.0-9.9 \%$ and a body mass index (BMI) of 


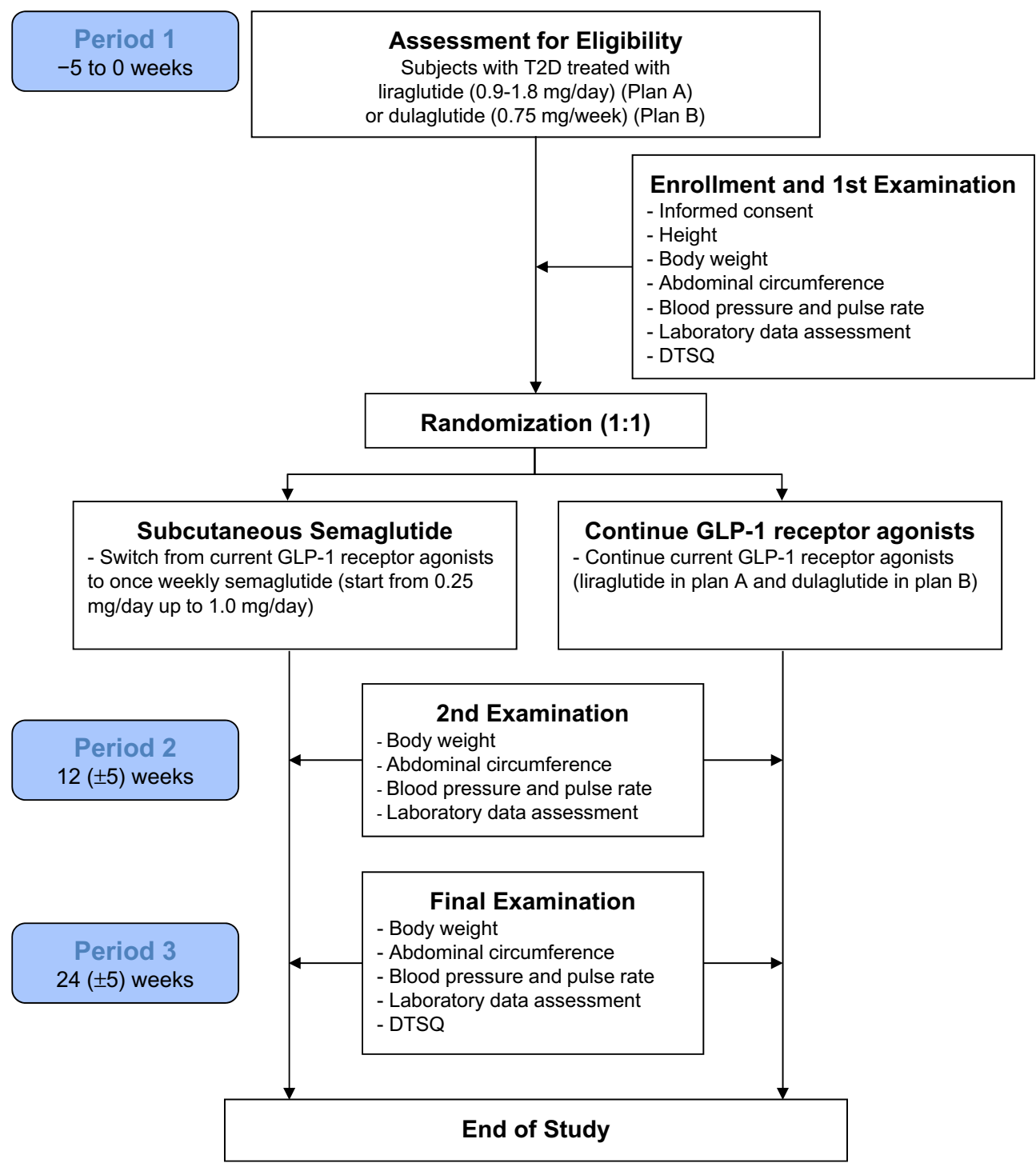

Fig. 1 Patient recruitment scheme. Participants will be randomly assigned to a group in which they continue to use their existing GLP-1 receptor agonist or another group in which they are switched to once-weekly semaglutide (starting dose $0.25 \mathrm{mg} /$ week). All the participants will

$\geq 22 \mathrm{~kg} / \mathrm{m}^{2}$, and have been treated with liraglutide (plan A: 0.9-1.8 mg/day) or dulaglutide (plan B: $0.75 \mathrm{mg} /$ week) for $\geq 12$ weeks (see Box 1). Participant enrollment will take place between November 6th, 2020 and December 31st, 2023 at seven medical centers located in Hokkaido. The inclusion and exclusion criteria for the trial are listed in Boxes 1 and 2. undergo DTSQ assessments at baseline and the end of the study. DTSQ Diabetes Treatment Satisfaction Questionnaire, GLP-1 glucagon-like peptidase-1, T2D type 2 diabetes

A clinician in the research team will obtain written informed consent from all eligible participants. The written material, consisting of a participant information leaflet and consent documentation, has been approved by the Research Committee. There will be an opportunity for the participants to freely ask members of the research team questions, and they will be able to withhold their consent at any time 
during the study period should they so wish. Patients will stop participating in the trial if any of the following criteria apply: (1) consent is withdrawn, (2) the physician decides-on the basis of the patient's condition-that the patient should stop participating, (3) the study is discontinued, or (4) the physician decides that the patient should stop participating for another reason.

Randomization will be carried out centrally using a web-based system (NorthNet). Given that the adequacy of glycemic control before randomization can affect the efficacy of the treatment, all participants will be randomly assigned at a ratio of 1:1 to either continue their existing GLP-1 receptor agonist or switch to semaglutide according to their age, BMI, and HbA1c level. The strategies that will be employed to improve compliance with the intervention protocol and the procedures for monitoring this compliance will be as follows: (1) repeated instruction in the use of devices for each GLP-1 receptor agonist at each clinic visit; (2) when patients attend the clinic with family members, the study will be explained to both the patient and his or her family; (3) each clinic will designate dedicated staff to explain the study protocol to patients and their families.

In plan $\mathrm{A}$, the sample size was calculated on the basis that semaglutide $(0.5 \mathrm{mg} /$ day $)$ will improve the HbA1c level by at least $0.81 \%$ (SD $0.77 \%)$ compared with liraglutide $(0.9 \mathrm{mg} /$ day $)$, as shown in phase III trials (each vs. placebo) and a network meta-analysis of the use of subcutaneous semaglutide in T2D patients $[17,18]$. In plan B, the sample size was calculated on the basis that semaglutide will improve HbA1c by at least $0.755 \% \quad$ (SD 1.00\%) compared with dulaglutide $(0.75 \mathrm{mg} /$ week $)$, as shown in the same trial and meta-analysis [17, 18]. Power calculations determined that a sample size of 16 individuals per group in plan A and 29 individuals per group in plan $B$ would be required to achieve a power of at least $80 \%$ to detect a difference between treatments. $P<0.05$ will be considered to represent statistical significance, and all tests will be two-sided. Based on the assumption that 2-3 participants (10\%) will drop out from each group in plans A and B, the sample size has been set at 18 participants per group in plan A and 32 in plan B. To ensure sufficient participant enrollment to achieve the target sample size, we will conduct the study at seven medical centers located in Hokkaido.

\section{Box 1: Inclusion Criteria}

- Japanese patients with T2D.

- Age 20-89 years.

- HbA1c 6.0-9.9\%.

- Body mass index $\geq 22 \mathrm{~kg} / \mathrm{m}^{2}$.

- Treatment with liraglutide or dulaglutide for at least 12 weeks before enrollment.

\section{Box 2: Exclusion Criteria}

- Treatment with any GLP-1 receptor agonist other than liraglutide or dulaglutide.

- Allergy to semaglutide.

- Unstable diabetic retinopathy.

- Current severe liver dysfunction or nephropathy.

- Severe infection, trauma, and/or recent or planned surgery.

- Severe ketosis.

- Diabetic coma or pre-coma.

- Pregnancy.

- Incompatibility with the trial for other reasons, as determined by the physician.

\section{Measurements and Data Collection}

The primary endpoint of the study is the change in $\mathrm{HbA1c}$ from baseline to week 24, which will be compared between the semaglutide and control groups. The secondary endpoints are as follows: the mean changes in (1) total DTSQ score, (2) body mass, (3) abdominal circumference, (4) systolic and diastolic BP, (5) pulse rate, (6) laboratory parameters indicative of glucose and lipid metabolism and liver and renal function, (7) factors associated with improvement in HbA1c and secondary endpoints, and (8) any side effects. The DTSQ includes eight items, and responses are scored on a seven-point scale from 0 to 6 . The scores for six items of the DTSQ (current treatment, convenience, flexibility, understanding, recommend, and continue) will be added together to give the overall treatment satisfaction score (range 0-36), with higher 
scores denoting greater treatment satisfaction. In addition, the perceived frequencies of hyperglycemia and hypoglycemia are assessed in the DTSQ, where they are rated on a scale of 0 ("never") to 6 ("most of the time"). Patients will complete a Japanese version of the DTSQ at baseline and during week 24 [19]. The total score and the score for each category obtained at each stage will be used for statistical analyses as a secondary endpoint. The relationships between the reduction in $\mathrm{HbA1c}$ and the changes in other parameters will also be assessed. We will prepare a time-course sheet for each study visit to minimize the risk of dropout.

\section{Data Analysis}

Analysis of the primary and secondary endpoint data will be performed using the full analysis set (FAS), which will comprise participants who are enrolled in the study and assigned to treatment groups. Patients who do not meet the inclusion criteria, for whom insufficient primary endpoint data are available, or who appreciably deviate from the study protocol will be excluded from the FAS. Differences between the two groups will be analyzed for statistical significance using the unpaired $t$ test or Mann-Whitney $U$ test for continuous data and Pearson's chi-square test or Fisher's exact test for categorical data. The factors associated with improvements in HbA1c and other metabolic factors will be assessed using analysis of covariance and multivariate analysis. We will analyze the data using JMP Pro v14.1.1 (SAS Institute, Cary, NC, USA), BellCurve for Excel (Social Survey Research Information Co., Ltd., Japan), and GraphPad Prism 8 v8.2.1 (GraphPad Software, Inc., San Diego, CA, USA).

\section{STRENGTHS AND LIMITATIONS}

To date, no studies have directly compared the efficacy of semaglutide with respect to glycemic control and treatment satisfaction with the efficacies of other GLP-1 receptor agonists in Asian T2D patients. A previous network metaanalysis revealed that the administration of semaglutide $0.5 \mathrm{mg}$ /week yielded greater $\mathrm{HbA} 1 \mathrm{c}$ and body mass reductions than liraglutide $1.2 \mathrm{mg} /$ day or lixisenatide [20]. However, because Asian patients with T2D tend to have lower BMIs than Western T2D patients, it is important to confirm this difference in efficacy in Asian populations.

GLP-1 receptor agonists that are available in clinical practice differ in their effects on glycemia and body weight, principally because of differences in duration of action. In general, short-acting GLP-1 receptor agonists are superior to long-acting agonists at reducing appetite, body weight, and postprandial glucose [21]. Semaglutide has been reported to have substantial effects on HbA1c and body weight, despite only being administered once weekly [15]. In the present trial there will be two arms: plan $\mathrm{A}$ is a comparison of semaglutide and liraglutide, and plan B is a comparison of semaglutide and dulaglutide. In plan A, we will assess the effect of semaglutide not only on glycemic control but also on body mass after the participants switch from daily liraglutide. Although it is known that long-acting GLP-1 receptor agonists have diminishing effects on gastric emptying over time, subcutaneous semaglutide is reported to continue to delay gastric emptying, implying that it is less affected by tachyphylaxis [22]. It will be important to confirm that similar effects occur in Asian people, whose BMIs are generally lower than those of Western T2D patients.

In contrast, plan B represents a comparison between weekly subcutaneous injectable therapies. Because the frequency of administration will be the same in plan $B$, this arm of the trial may reveal changes in QOL that are not associated purely with the injection frequency itself. The use of semaglutide is expected to improve satisfaction, even in this population, because good glycemic control itself has been shown to be associated with high patient satisfaction [23]. In addition, post-hoc subanalysis of the SUSTAIN trial data showed a significant improvement in overall treatment satisfaction when semaglutide was administered versus comparators or placebo [24]. It is worth mentioning that the participants in the present trial will switch from other GLP-1 receptor agonists to semaglutide, so we will be able to determine 
whether even such an apparently minor change can improve satisfaction. As stated above, semaglutide has been reported to delay gastric emptying, despite it being a long-acting drug. However, it may cause nausea more frequently than the other drugs, which may worsen QOL, so it will also be important to record side effects as well as QOL during the trial.

GLP-1 receptor agonists have pleiotropic effects (e.g., beta-cell protection, anti-inflammation, and cardioprotective effects) as well as a hypoglycemic effect $[25,26]$. Because semaglutide has been reported to have superior effects on glycemic control, we will also assess beta-cell function and atherosclerosis-related parameters, such as lipid profile and BP, in each group. Finally, it is worth mentioning that this study will be conducted in a standard real-world clinical practice setting.

In conclusion, the present study will be the first clinical trial to evaluate the efficacy of semaglutide for glycemic control and treatment satisfaction in Japanese patients with T2D who were previously being treated with other GLP-1 receptor agonists. The results of the study may therefore provide new insights into the pleiotropic effects of semaglutide in patients with T2D.

\section{ETHICS AND DISSEMINATION}

\section{Ethics Approval}

The trial was registered with the University Hospital Medical Information Network (UMIN) Center (UMIN000042369) and the Japan Registry of Clinical Trials (jRCT1011200008) before enrollment commenced. The study protocol (no. 018-005) was approved by the Hokkaido University Certified Review Board (CRB no. 1180001), and the current version is 6.0 (approved on October 8th, 2020). The study will be carried out according to the principles of the Declaration of Helsinki and its amendments.

\section{Data Protection and Management}

Data management, including coding, security, storage, and cleaning, will be performed by researchers throughout the trial. The study data will be archived at Hokkaido University for 5 years after study completion or 3 years after disclosure of the study results. Participants will also be able to obtain the final results of the study. The UMIN and jRCT databases will contain detailed information regarding the study. Study conduct will be evaluated by a monitor who will be independent of the investigators. Monitoring will be performed on the first and fifth participants at Hokkaido University Hospital, and on the first participant at each of the other study sites. In line with the Clinical Trials Act in Japan, adverse events and other information, including modification of the trial, will be reported and disclosed publicly.

\section{ACKNOWLEDGEMENTS}

We also thank the participants in the study.

Funding. No funding or sponsorship was received for this study or the publication of this article. The Rapid Service Fee was funded by the authors.

Editorial Assistance. We thank Mark Cleasby, PhD, from Edanz Group (https://enauthor-services.edanzgroup.com/ac) for editing a draft of this manuscript. This was funded by the authors.

Authorship. All named authors meet the International Committee of Medical Journal Editors (ICMJE) criteria for authorship for this article, take responsibility for the integrity of the work as a whole, and have given their approval for this version to be published.

Authorship Contributions. C.O. and H.M. designed the original study protocol. H.N., Y.T., and K.Y.C. modified the study design. H.N. and H.M. drafted the manuscript, and all the other authors helped to revise it. All authors will 
contribute to participant enrollment. H.N. and Y.T. will collect the data and contribute to statistical analysis. H.M. is the guarantor of this work and will take responsibility for the integrity of the data and the accuracy of the data analysis.

Disclosures. S.N., Y.K., A.N., T.A., and H.M. have received honoraria for lectures and received research funding from some organizations, as described below. S.N. has received honoraria for lectures from Eli Lilly, Sumitomo Dainippon Pharma Co., and Novartis Pharma. Y.K. has received honoraria for lectures from AstraZeneca, Mitsubishi Tanabe Pharma Co., Ltd., MSD, Ono Pharmaceutical Co., Ltd., Sanofi, Taisho Pharmaceutical Co., Ltd., Kowa Pharmaceutical Co. Ltd., Eli Lilly, Sumitomo Dainippon Pharma Co., and Takeda Pharmaceutical Co., Ltd. A.N. has obtained research support from Mitsubishi Tanabe Pharma Co., Daiichi Sankyo Co. Ltd., MSD, Novo Nordisk Pharma, Novartis Pharma, AstraZeneca, LifeScan Japan, and Taisho Pharmaceutical. T.A. has received honoraria for lectures from Mitsubishi Tanabe Pharma Co., Chugai Pharmaceutical Co., Ltd., Astellas Pharma Inc., Takeda Pharmaceutical Co., Ltd., Pfizer Inc., AbbVie Inc., Eisai Co. Ltd., Daiichi Sankyo Co. Ltd., BristolMyers Squibb Co., UCB Japan Co. Ltd., Eli Lilly Japan K.K., and has received research funding from Astellas Pharma Inc., Takeda Pharmaceutical Co., Ltd., Mitsubishi Tanabe Pharma Co., Chugai Pharmaceutical Co., Ltd., Daiichi Sankyo Co. Ltd., Otsuka Pharmaceutical Co., Ltd., Pfizer Inc., and Alexion Inc. H.M. has received honoraria for lectures from Astellas Pharma Inc., AstraZeneca, Sumitomo Dainippon Pharma Co., Eli Lilly, Kissei, Mitsubishi Tanabe Pharma Co., MSD, Novartis Pharma, Novo Nordisk Pharma, Takeda Pharmaceutical Co., Ltd., Kowa Pharmaceutical Co., Ltd., Ono Pharmaceutical Co., Ltd., and Sanofi; and has received research funding from Astellas Pharma Inc., AstraZeneca, Daiichi Sankyo Co. Ltd., Sumitomo Dainippon Pharma Co., Eli Lilly, Mitsubishi Tanabe Pharma Co., MSD, Novo Nordisk Pharma, Sanofi, Takeda Pharmaceutical Co., Ltd., Kowa Pharmaceutical Co., Ltd., Ono Pharmaceutical Co., Ltd., and Taisho Toyama
Pharmaceutical Co., Ltd. H.N., C.O., Y.T., J.T., H.Y., S.T., S.A., H.K., and K.Y.C. have nothing to disclose.

Data Availability. The datasets generated during and/or analyzed during the current study are available from the corresponding author on reasonable request.

Open Access. This article is licensed under a Creative Commons Attribution-NonCommercial 4.0 International License, which permits any non-commercial use, sharing, adaptation, distribution and reproduction in any medium or format, as long as you give appropriate credit to the original author(s) and the source, provide a link to the Creative Commons licence, and indicate if changes were made. The images or other third party material in this article are included in the article's Creative Commons licence, unless indicated otherwise in a credit line to the material. If material is not included in the article's Creative Commons licence and your intended use is not permitted by statutory regulation or exceeds the permitted use, you will need to obtain permission directly from the copyright holder. To view a copy of this licence, visit http://creativecommons.org/licenses/by$\mathrm{nc} / 4.0 /$.

\section{REFERENCES}

1. Gaede P, Lund-Andersen H, Parving HH, Pedersen O. Effect of a multifactorial intervention on mortality in type 2 diabetes. N Engl J Med. 2008;358: 580-91.

2. Sone $\mathrm{H}$, Tanaka S, Ishibashi $\mathrm{S}$, et al. The new worldwide definition of metabolic syndrome is not a better diagnostic predictor of cardiovascular disease in Japanese diabetic patients than the existing definitions: additional analysis from the Japan Diabetes Complications Study. Diabetes Care. 2006;29:145-7.

3. Ueki K, Sasako T, Okazaki Y, et al. Effect of an intensified multifactorial intervention on cardiovascular outcomes and mortality in type 2 diabetes (J-DOIT3): an open-label, randomised controlled trial. Lancet Diabetes Endocrinol. 2017;5:951-64. 
4. UK Prospective Diabetes Study (UKPDS) Group. Intensive blood-glucose control with sulphonylureas or insulin compared with conventional treatment and risk of complications in patients with type 2 diabetes (UKPDS 33). Lancet. 1998;352: 837-53.

5. Khunti K, Davies M, Majeed A, Thorsted BL, Wolden ML, Paul SK. Hypoglycemia and risk of cardiovascular disease and all-cause mortality in insulin-treated people with type 1 and type 2 diabetes: a cohort study. Diabetes Care. 2015;38: 316-22.

6. Rossi MC, Nicolucci A, Ozzello A, et al. Impact of severe and symptomatic hypoglycemia on quality of life and fear of hypoglycemia in type 1 and type 2 diabetes. Results of the Hypos-1 observational study. Nutr Metab Cardiovasc Dis. 2019;29:736-43.

7. Charpentier G, Fleury F, Dubroca I, Vaur L, Clerson P. Electronic pill-boxes in the evaluation of oral hypoglycemic agent compliance. Diabetes Metab. 2005;31:189-95.

8. Miya A, Nakamura A, Miyoshi H, et al. Satisfaction of switching to combination therapy with lixisenatide and basal insulin in patients with type 2 diabetes receiving multiple daily insulin injection therapy: A randomized controlled trial. J Diabetes Investig. 2018;9:119-26.

9. Nauck M. Incretin therapies: highlighting common features and differences in the modes of action of glucagon-like peptide-1 receptor agonists and dipeptidyl peptidase- 4 inhibitors. Diabetes Obes Metab. 2016;18:203-16.

10. Drucker DJ. The cardiovascular biology of glucagon-like peptide-1. Cell Metab. 2016;24:15-30.

11. Nauck MA, Kemmeries G, Holst JJ, Meier JJ. Rapid tachyphylaxis of the glucagon-like peptide 1-induced deceleration of gastric emptying in humans. Diabetes. 2011;60:1561-5.

12. Kristensen SL, Rørth R, Jhund PS, et al. Cardiovascular, mortality, and kidney outcomes with GLP-1 receptor agonists in patients with type 2 diabetes: a systematic review and meta-analysis of cardiovascular outcome trials. Lancet Diabetes Endocrinol. 2019;7:776-85.

13. Bethel MA, Patel RA, Merrill P, et al. Cardiovascular outcomes with glucagon-like peptide-1 receptor agonists in patients with type 2 diabetes: a metaanalysis. Lancet Diabetes Endocrinol. 2018;6: 105-13.

14. Takase T, Nakamura A, Yamamoto C, et al. Improvement in treatment satisfaction after switching from liraglutide to dulaglutide in patients with type 2 diabetes: a randomized controlled trial. J Diabetes Investig. 2019;10:699-705.

15. Marso SP, Bain SC, Consoli A, et al. Semaglutide and cardiovascular outcomes in patients with type 2 diabetes. N Engl J Med. 2016;375:1834-44.

16. Bradley C. The Diabetes Treatment Satisfaction Questionnaire: DTSQ. In: Bradley C, editor. Handbook of psychology and diabetes: a guide to psychological measurement in diabetes research and practice. Chur: Harwood Academic; 1994. pp. 111-132.

17. Odawara M, Miyagawa J, Iwamoto $\mathrm{N}$, Takita $\mathrm{Y}$, Imaoka T, Takamura T. Once-weekly glucagon-like peptide-1 receptor agonist dulaglutide significantly decreases glycated haemoglobin compared with once-daily liraglutide in Japanese patients with type 2 diabetes: 52 weeks of treatment in a randomized phase III study. Diabetes Obes Metab. 2016;18: 249-57.

18. Webb N, Orme M, Witkowski M, Nakanishi R, Langer J. A network meta-analysis comparing semaglutide once-weekly with other GLP-1 receptor agonists in Japanese patients with type 2 diabetes. Diabetes Ther. 2018;9:973-86.

19. Ishii H, Bradley $\mathrm{C}$, Riazi A, Barendse $\mathrm{S}$, Yamamoto T. The Japanese version of the Diabetes Treatment Satisfaction Questionnaire (DTSQ): translation and clinical evaluation [published in Japanese]. J Clin Exp Med. 2000;192:809-14.

20. Witkowski M, Wilkinson L, Webb N, Weids A, Glah D, Vrazic H. A systematic literature review and network meta-analysis comparing once-weekly semaglutide with other GLP-1 receptor agonists in patients with type 2 diabetes previously receiving 1-2 oral anti-diabetic drugs. Diabetes Ther. 2018;9: 1149-67.

21. Meier JJ. GLP-1 receptor agonists for individualized treatment of type 2 diabetes mellitus. Nat Rev Endocrinol. 2012;8:728-42.

22. Hjerpsted JB, Flint A, Brooks A, Axelsen MB, Kvist T, Blundell J. Semaglutide improves postprandial glucose and lipid metabolism, and delays first-hour gastric emptying in subjects with obesity. Diabetes Obes Metab. 2018;20:610-9.

23. Moreira ED Jr, Neves RC, Nunes ZO, et al. Glycemic control and its correlates in patients with diabetes in Venezuela: results from a nationwide survey. Diabetes Res Clin Pract. 2010;87:407-14.

24. Jendle J, Birkenfeld AL, Polonsky WH, et al. Improved treatment satisfaction in patients with type 2 diabetes treated with once-weekly 
semaglutide in the SUSTAIN trials. Diabetes Obes Metab. 2019;21:2315-26.

25. Aroda VR. A review of GLP-1 receptor agonists: Evolution and advancement, through the lens of randomised controlled trials. Diabetes Obes Metab. 2018;20(Suppl 1):22-33.

26. Drucker DJ. Mechanisms of action and therapeutic application of glucagon-like peptide-1. Cell Metab. 2018;27:740-56. 